\title{
Specialist services and medical training for the care of elderly people with mental illness
}

This paper is a summary of a report published in February 1989 by the Royal Colleges of Physicians (of London) and Psychiatrists*. It was produced by a joint working party set up to review progress and problems in the provision of specialist and hospital care to this expanding client group. The report traces the development of specialist services for the psychiatry of old age, outlines the main features and working relationships of such services and considers the implications for general and specialist medical training. The working party has also initiated a research project to examine the scope and effectiveness of different models of service for elderly people with mental illness.

\section{Growth of specialisation in old age psychiatry}

During the late 1960 s, concern over the organisation of care for elderly people with mental illness, and particularly those with dementia, led to efforts by the DHSS to promote collaboration between psychiatrists, geriatricians and social services departments. Although the joint psychogeriatric assessment unit, seen at that time as the focal point of this collaboration, never really caught on, in the last 15-20 years there has been a dramatic rise in the number of psychiatrists opting to specialise in the care of the elderly (from a handful in 1970 to over 250 by 1985). Cooperation with geriatric medicine has usually advanced as a result.

This trend has derived mainly from local initiative and enthusiasm with marked regional variation possibly reflecting the impact of some of the pioneers in this field. Despite important contributions, such as the Health Advisory Service publication The Rising Tide and the government initiative linked with it, the response in terms of central policy has been somewhat tentative. There has also been a dearth of official data on psychiatric services for the elderly. There is now wide agreement on the benefit to old people (with functional illnesses as well as dementia) of such specialised services and on the resources these require. Many services, however, are seriously under resourced. Almost $30 \%$ of the elderly people in Britain were, in 1985, without access to such specialist care. The present shortfall in consultant posts in old age psychiatry is estimated at just over 200 .

*The full report is available from the College's Publications Department, price $£ 5$.
Many of the problems in obtaining information on facilities and in earmarking resources for the psychiatry of old age reflect the fact that it is not yet designated as an official specialty within psychiatry. Consideration of its current and proposed consultant numbers, the specific service patterns and training requirements and the accumulated body of knowledge and research has led the working party to conclude that such specialty status is now warranted; furthermore this recognition would be essential to future development and decisions on resource allocation and training.

\section{The district psychogeriatric service}

The report devotes some detail to the actual working of a psychogeriatric service with emphasis on those factors conducive to good practice. The range of caseload is agreed to include the full spectrum of psychiatric disorders presenting from the community among the over-65s and appropriate cases of presenile dementia. Emphasis is given to the deployment of staff in assessment, treatment and support of patients and their families in the home setting, and to the important bridging role of the community psychogeriatric nurse. This community care has to be backed up by an active use of short-stay beds and day hospitals balanced by a selective use of long-stay beds for very frail or difficult patients (with emphasis on a 'nursing home' style replacing the traditional custodial approach).

The changes in workload and resource needs generated by this more active approach (particularly in the often hitherto neglected area of functional illness in old people) and the effects of demographic change are discussed with reference to the shortcomings of existing norms; alternative figures are proposed. Reference is also made to the Royal College of Psychiatrists' recent 'Guidelines for Regional Advisers on Consultant Posts in the Psychiatry of old Age' (which stipulate minimum rather than desirable resource levels).

The relationships between the psychogeriatric service and other agencies involved in the care of the elderly are also discussed. Close links and harmony with geriatric medicine are essential, although not yet always achieved. The trend towards joint activity at a clinical and planning level is welcomed, as is the setting up in some centres of joint departments of health care for the elderly. Consideration is also given to the 
interfaces with other hospital specialties, primary medical care, social services departments and the burgeoning private and voluntary sectors. The role of the psychogeriatric service in support with difficult cases, in enhancing the skills of care staff with the elderly mentally ill and in monitoring standards of care, is particularly stressed. In this general context the working party's report was produced after the publication of the Griffiths' report on Community Care but before the government's response was known.

\section{Medical training in old age psychiatry}

Although psychogeriatricians play a part in teaching in all the British medical schools, the extent and quality of clinical and academic exposure of medical students to old age psychiatry varies widely and a number of schools still have no academic post in this field. Such a focus is clearly needed to stimulate and monitor the teaching of this subject, to co-ordinate it with that in geriatric medicine and to counteract the negative view of old age and its disorders often prevalent at this stage in medical training.

Psychogeriatric placements play a valuable part in many vocational training schemes for general practice. There is also a strong case for greater availability of such experience at the stage of general professional training for doctors aiming at a career in hospital medicine. This is relevant to the work of most specialties and there is also a need to facilitate sampling of this field (in which a substantial amount of recruitment will be necessary over the next few years).

Since 1981, trainees in psychiatry have been required to spend a 'significant proportion of time' in a specialised psychogeriatric service during preparation for membership of the Royal College of Psychiatrists. If old age psychiatry becomes a speciality in its own right this requirement might lapse. Nevertheless the value of such experience (as of child psychiatry or psychotherapy) in producing a fully rounded clinician and the need for trainees to sample this future career option would favour retaining its prominence among the options in registrar rotations.

The report discusses the present anomalies in training arrangements in old age psychiatry at senior registrar level. There are insufficient posts to provide the output necessary for the existing rate of consultant vacancies in this field, a problem accentuated by the rather low yield from such posts when incorporated in general psychiatry rotations. On the other hand, it is difficult to provide a satisfactory training in general and old age psychiatry concurrently in a single holder post. Experience in geriatric medicine should clearly be included in the training of prospective psychogeriatricians but is not yet a formal requirement. The retention of old age psychiatry within the specialty of general adult psychiatry would remain a major handicap to the development of a formal training programme at senior registrar level and to the expansion of training opportunities that will be essential for the consultant recruitment already identified.

\section{A research proposal}

There have been a number of published accounts of individual psychogeriatric services. These have inevitably reflected local anomalies and idiosyncracies which may have hindered their translation to other settings. In this report an attempt has been made to draw out the ingredients which seemed to warrant general application. The working party considers, however, that there is a need for evaluation of different models of service in different district contexts. Supported by generous grants from Marks and Spencer Plc and the Mental Health Foundation, the working party has initiated a research project along these lines for which it has been fortunate to engage the services of Miss Alison Norman whose work in this field is already well respected. Ten health districts have been selected representing a range of type and level of psychogeriatric service in different settings (in terms of demography, environment and hospital bases). Managers and staff of the psychogeriatric service are being interviewed about their concept of their roles, the service they provide, the resources available to them and their relations with other services. This will be complemented by interviews with 'consumers' (patients, carers and professionals from other agencies). The results will be presented as a series of case studies.

\section{Recommendations}

\section{Service developments}

(1) Old age psychiatry should be officially recognised as a specialty within psychiatry. This status will facilitate many of the recommendations which follow, and is justified by the number of consultants now working in this field, the specific service patterns, the training requirements and the body of knowledge and research.

(2) Every Health District should include a specialised service for the psychiatry of old age based on the model outlined in this report and backed by at least the minimum resources set out in the Royal College of Psychiatrists Guidelines.

(3) An effective old age psychiatry service requires specialist consultant cover of one session for every 2,000 elderly people (with enhancement in teaching districts). Achievement nationally of such cover (which already exists in many districts) would entail the present annual 
recruitment of 25-30 (WTE) consultants to this field being maintained for the next ten years.

(4) Resources, workload, manpower and training facilities relating to old age psychiatry should be clearly and easily identified and the data used by the DHSS and the regions to ensure that policies are effectively implemented in what has long been recognised as a priority area.

(5) Improved collaboration between geriatric medicine and old age psychiatry should be constantly pursued since this is essential for good patient care, efficient use of resources and effective planning and liaison with other services. Joint departments, should be more widely considered.

(6) Members of psychogeriatric teams should be actively involved in supporting, observing and training staff engaged in providing alternative forms of domiciliary and residential care. However, skilled long-term hospital care must remain available for patients with severe behaviour problems.

\section{Education, training and research}

(7) Each medical school should have an adequately supported senior academic post in old age psychiatry to develop and evaluate teaching for medical students and allied professions and to promote research in this field.

(8) All medical students should receive training in old age psychiatry in approved specialised departments with clinical exposure in both hospital and community. Consideration should be given to integrating such teaching with that in geriatric medicine.

(9) Posts in old age psychiatry (in conjunction with geriatric medicine or general psychiatry) should be included among the options for the general professional level of postgraduate medical training for doctors aiming at a career in hospital medicine, as well as for those training for general practice.

(10) Those intending to practise general psychiatry should be encouraged to spend a period at some stage of their training in approved departments of old age psychiatry. The clinical experience should reflect the full range of the service and should be geared to training as opposed to service needs.

(11) Higher professional training for specialisation in the psychiatry of old age should include: two years in old age psychiatry, at least two months experience in geriatric medicine, further experience in general psychiatry.

(12) Where higher training for the psychiatry of old age is incorporated in Regional general senior registrar rotations, such schemes should be monitored to ensure that the appropriate output of psychogeriatricians is achieved. This applies particularly to the posts recently allocated by JPAC $^{2}$ to enhance training opportunities in this field, the uptake and targeting of which has been in some doubt.

(13) Guidelines should be agreed between the respective higher training committees as to the experience and training required by senior registrars in geriatric medicine and old age psychiatry in each other's specialty. They should also uphold the supernumerary concept of the senior registrar grade in respect of the workload implications of such secondment. Mutual exchanges and linked training in joint departments should be encouraged.

(14) There is a need for continuing evaluative research in the psychiatry of old age (including models of collaboration with geriatric medicine and other services). It is hoped that the research project initiated by this working party will contribute to this process.

\section{Reference}

Royal College of Psychiatrists (1987) Guidelines for Regional Advisers on Consultant Posts in the Psychiatry of old Age. Bulletin of the Royal College of Psychiatrists, 11, 240-242.

'(WTE $)=$ Whole time equivalent

${ }^{2}($ JPAC $)=$ Joint Planning Advisory Committee on Manpower

\section{'The Practical Administration of Electroconvulsive Therapy (ECT)'}

Council has recently approved a report of the ECT Sub-Committee of the Research Committee entitled The Practical Administration of Electroconvulsive Therapy (ECT). It has been agreed that each ECT Suite should have a complimentary copy of this report. Consultants in charge of ECT should therefore write to the Publications Department at the College enclosing an A4 self-addressed envelope and 26p in stamps.

VANESSA CAMERON The Secretary 